\title{
Erratum to: Rapid microsatellite marker isolation for the Pink Stem Borer, Sesamia inferens (Lepidopetera: Noctuidae) through 454 GS-FLX Titanium pyrosequencing of enriched DNA libraries and cross amplification in related taxa
}

\author{
Yudi Liu $\cdot$ Xingkui An $\cdot$ Maolin Hou
}

Published online: 14 November 2014

(c) The Japanese Society of Applied Entomology and Zoology 2014

Erratum to: Appl Entomol Zool

DOI 10.1007/s13355-014-0286-7

Unfortunately, the author names were incorrectly published in the original publication. The author group should appear as "Yudi Liu · Xingkui An · Maolin Hou" instead of "Liu Yudi · An Xingkui · Hou Maolin".

The online version of the original article can be found under doi:10.1007/s13355-014-0286-7.

Y. Liu $\cdot$ X. An $\cdot$ M. Hou $(\bowtie)$

State Key Laboratory for Biology of Plant Diseases and Insect

Pests, Institute of Plant Protection, Chinese Academy

of Agricultural Sciences, Beijing 100193, People's Republic

of China

e-mail:mlhou@ippcaas.cn 